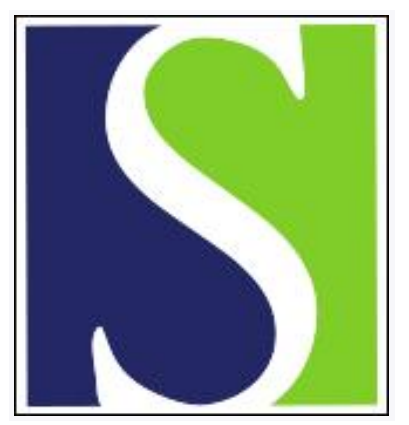

Scand J Work Environ Health 2012;38(2):144-154

https://doi.org/10.5271/sjweh.3199

Published online: 10 Oct 2011, Issue date: Mar 2012

Breast cancer survivors' views of factors that influence the return-to-work process - a qualitative study

by Tamminga SJ, de Boer AGEM, Verbeek JHAM, Frings-Dresen MHW

Affiliation: Coronel Institute of Occupational Health/Academic Medical Center, University of Amsterdam, PO Box 22700, 1100 DE Amsterdam, the Netherlands. S.J.Tamminga@amc.uva.nl

The following articles refer to this text: 2019;45(4):370-375;

2020;46(2):177-187

Key terms: breast cancer; cancer; experience; oncology; qualitative research; return to work; return-to-work; RTW

This article in PubMed: www.ncbi.nlm.nih.gov/pubmed/21986836

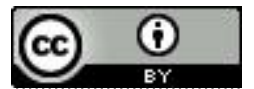




\title{
Breast cancer survivors' views of factors that influence the return-to-work process - a qualitative study
}

\author{
by Sietske J Tamminga, MSc, ${ }^{1}$ Angela GEM de Boer, PhD, ${ }^{1}$ Jos HAM Verbeek, MD, PhD, ${ }^{1,2}$ Monigue HW \\ Frings-Dresen, PhD $^{1}$
}

\begin{abstract}
Tamminga SJ, de Boer AGEM, Verbeek JHAM, Frings-Dresen MHW. Breast cancer survivors' views of factors that influence the return-to-work process - a qualitative study. Scand J Work Environ Health. 2012;38(2):144-154. doi:10.5271/sjweh.3199
\end{abstract}

Objectives Accumulating evidence suggests that most employed breast cancer survivors are able to return to work but often experience difficulties in the process. The objective of this study was to identify: (i) factors experienced as barriers to and facilitators of the return-to-work (RTW) process, (ii) which factors were important during initial and post RTW, and (iii) possible solutions to RTW problems.

Methods Twelve breast cancer survivors participated in semi-structured interviews. Interviews were thematically analyzed using MAXQDA, software for qualitative data analysis. We used the World Health Organization's International Classification of Functioning, Disability and Health as a conceptual framework.

Results Participants experienced many barriers to and facilitators of RTW. In line with previous studies, we found that work environmental factors, such as support from a supervisor, importance of work, and physical or psychological side-effects (such as fatigability), influenced RTW. In addition, we found that barriers included temperament and personality functions, "job lock", and societal attitudes, while facilitators comprised taking care of one's health, skills/coping, and support from family and healthcare professionals. During the initial RTW phase, physical or psychological side-effects hampered work resumption, while during the post RTW phase, a lack of understanding from the work environment was problematic. Participants mentioned that guidance from healthcare professionals and information for supervisors and colleagues should be improved.

Conclusions To enhance RTW among breast cancer survivors, interventions should focus on barriers and facilitators for individuals at different time points in the RTW process. Better guidance from healthcare professionals and information for supervisors and colleagues could also enhance the process.

Key terms experience; oncology; return to work; RTW; qualitative research.

Accumulating evidence suggests that most employed breast cancer survivors are able to return to work $(1-3)$ but often experience difficulties managing their work, for instance due to physical (eg, arm function) or cognitive work limitations (eg, concentration, fatigue), or unreasonable treatment at the workplace (eg, feelings of being discriminated against, unsupportive work environment) (4-6). It is important to understand the difficulties cancer survivors experience in order to develop interventions to better support them in their resumption of work.

Using regression analysis based on self-report questionnaires or administrative data, many previous studies have shown that several factors can enhance or hinder the return to work (RTW) of cancer survivors $(3,7-11)$. However, the difficulties of managing work depend on a complex interaction of individual and environmental factors and the social security system $(4,12)$, and these difficulties may change during the course of the disease. This underlying context may not be identified by outcomes based on questionnaires or administrative data. Furthermore, RTW is often defined in quantitative studies as a singular event, but it can also be viewed as a process during which different factors influence RTW in different phases, such as the initial and the post RTW phase (13). Therefore, qualitative research of the initial and post RTW processes may be a better method for

1 Coronel Institute of Occupational Health, Academic Medical Center, University of Amsterdam, Amsterdam, the Netherlands.

2 Finnish Institute of Occupational Health Field, Kuopio, Finland.

Correspondence to: Sietske J Tamminga, Coronel Institute of Occupational Health/Academic Medical Center, University of Amsterdam, PO Box 22700, 1100 DE Amsterdam, the Netherlands. [E-mail: S.J.Tamminga@amc.uva.nl] 
studying this problem. In the international literature, a number of studies have applied this qualitative research method by analyzing breast cancer survivors' experiences with the RTW process (6).

This study aims to add to these previous qualitative studies by (i) identifying factors that have been experienced as barriers to or facilitators of the initial and post RTW processes, (ii) classifying these factors according to the World Health Organization's International Classification of Functioning, Disability and Health (ICF) (14), and (iii) asking cancer survivors for possible solutions to their RTW problems. The ICF is a framework to describe health and disability and their impact on functioning. The ICF consists of the following domains: disease/disorder, body function, body structure, activity/ participation, and personal and environmental factors (14). We chose the ICF as a conceptual framework for this study because it allows a comprehensive analysis of the factors influencing RTW.

RTW of sick-listed employees is influenced by the institutional context of the social security system of a country. In the Netherlands, the employee cannot be fired due to his/her illness during the first two years of sickness absence and receives at least $70 \%$ of their wage, which is paid by the employer. Both the employer and the employee have responsibility for the RTW process.

\section{Method}

The study was designed to be qualitative, using semistructured interviews. We used the items of the consolidated criteria for reporting qualitative research (COREQ) for improving the quality of reporting qualitative research (15). We sought ethical approval for this study from the Medical Ethics Committee of the Academic Medical Centre, who judged that ethical approval was not required. All interviews were held between May and July 2010.

\section{Participant selection}

Cancer survivors were eligible to participate if they were: (i) primarily diagnosed with breast cancer, (ii) between 18-65 years of age, (iii) working at the time of diagnosis, and (iv) fluent in Dutch. We refer to cancer survivors as individuals who have been diagnosed with cancer and are still living. Cancer survivors were recruited via a notice that was posted on the websites of three Dutch cancer patient organizations, where cancer survivors could register for participation. Thereafter, the interviewer contacted them by telephone to check eligibility and explain the aim and the content of the study. The first ten consecutive breast cancer survivors who registered were enrolled in the study. Thereafter, a sampling approach was conducted with several breast cancer survivors who registered but were not yet enrolled in the study in order to capture various experiences and working situations. This sampling approach was conducted based on the type of contract and RTW phase.

\section{Interview procedure}

Before the start of the face-to-face interview, each participant was explained the purpose of the study, the position of the interviewer, and the fact that information was handled confidentially. Each participant signed an informed consent form before the start of the interview. All interviews were audio-recorded. Participants were asked to fill in a questionnaire to assess participant (eg, age), disease (eg, treatment type), and work-related characteristics (eg, occupation, importance of work measured on a visual analogue scale) and to prepare participants for the topics of the interview (eg, facilitators of and barriers to RTW).

Semi-structured interviews were centered on a topic list. The topic list was generated on the basis of the literature and expertise of the research team and was pilot tested with an experienced interviewer and a cancer survivor. The topic list consisted of four items: (i) understanding the diagnosis, treatment, and work situation (pre-diagnosis and current); (ii) identifying the factors that were barriers to and facilitators of the initial and post RTW process; (iii) identifying if factors that were retrieved non-systematically from the literature $(4,12,16-19)$ were experienced as barriers to or facilitators of RTW if not already mentioned during the interview; and (iv) identifying possible solutions to RTW problems. The interviewer started with open-ended questions and subsequently asked more specific in-depth questions. Participants were encouraged to explain in detail how they experienced each factor. The interviewer summarized all mentioned factors so that participants could indicate if something was misunderstood or missing. All participants were interviewed once only, face-to-face, without anyone else present because that might have biased the participants' viewpoint or inhibited the discussion. The interviews were carried out at a place the participant preferred. All interviews were carried out by the first author, who is a female $\mathrm{PhD}$ student currently working on the topic of cancer survivors and RTW. The interviewer did not have a direct relationship with the participants and did not represent an organization or a healthcare profession involved in cancer or the RTW care of cancer survivors.

\section{Statistical analysis}

The interviews were transcribed verbatim. Neither the transcripts nor analysis of the transcripts were returned 
to participants for comments or feedback. One of the authors analyzed the factors that were mentioned during the interview after each interview to record if new factors were mentioned. We stopped interviewing when no new factors were mentioned. The transcripts were coded using the MAXQDA (Verbi GmbH, Marburg, Germany) qualitative data analysis software package.

First, the transcripts were read to become familiar with the content of the interviews. Then, they were opencoded in the same order as the interviews were held. The goal of open-coding was to identify all important aspects of the text that answered the research question. The labels of the open codes represented the text as closely as possible. In addition, these open codes were classified into barriers and facilitators and generally classified in the context of the ICF (ie, health-related, personal, and environmental). Finally, the open codes were classified according to the ICF (ie, disease/disorder, body functions/body structure, activity/participation, personal factors, and environmental factors) using the core set for breast cancer $(20)$ and vocational rehabilitation $(21,22)$ as a starting point. These factors were predetermined by the ICF unless such a factor could not be identified (eg, personal factors). Each core set consists of a set of ICF factors that are relevant to a specific condition and were formulated by experts. Furthermore, we used the classification of work-related factors as described by Minis et al (23). In accordance with the ICF, we categorized unchangeable temperament and personality functions as body functions and changeable temperament and personality functions as personal factors. Finally, we identified which factors were considered by participants as the key barriers to or facilitators of their RTW.

In a separate analysis, factors that were important during the initial and post RTW processes and possible solutions to barriers were identified and labeled. Initial RTW was defined as the period from diagnosis until returning to work. Post RTW was defined as the period from initial to sustainable RTW.

To improve inter-observer reliability, another researcher coded two interviews independently and discussed the results until consensus was reached. Furthermore, before each step in the analysis took place, all authors discussed the content in order to reach consensus. Factors that did not affect RTW but did affect, for example, work productivity or quality of work life were not taken into consideration.

\section{Results}

All the breast cancer survivors who registered were eligible to participate. The interviewer contacted them by telephone to explain the content of the study, after which all the breast cancer survivors agreed to participate. After 12 interviews, no new factors were retrieved from the interviews. Nine interviews took place at the participant's homes, two at the hospital, and one at the participant's workplace. During the interviews, no one else was present, except the spouse of participant E during the last 20 minutes. The duration of the interviews ranged from 40-75 minutes.

\section{Participant characteristics}

The mean age of participants was 42 [standard deviation (SD) 7] years at the time of the interview. Participants had different social backgrounds and educational levels but had the same Dutch cultural background.

\section{Disease-related characteristics}

Primary diagnosis of breast cancer was made on average two years before the interview (table 1). Two participants had a relapse of breast cancer and were diagnosed with metastatic cancer. All participants underwent surgery, ten received chemotherapy, nine radiotherapy, and six hormone treatment. At the time of the interview, six participants were still receiving treatment, while the others had ended treatment between nine months and four years prior.

\section{Work-related characteristics}

The participants had different types of occupations and contracts before diagnosis and at the time of the interview (table 2). Five participants had altered their occupation after cancer diagnosis either due to altered work interest or because it was not possible to carry out their former occupation due to job loss or side-effects of cancer treatment (eg, fatigue, cognitive problems). Two participants had an improved work situation, and six had a poorer work situation, two of whom had the prospect of improvement in the near future. Six participants were (partly) on paid sick leave or (partly) on disability pension at the time of the interview. Participants rated the importance of work on a visual analogue scale of $0-100$ with a median of 66 (range 10-85). Three participants were solely the breadwinner, six participants contributed equally with their husband to the family income, and in three cases the husband was the breadwinner.

\section{Return-to-work process}

All participants had a different complex pattern of RTW. The time from initial sick leave to sustainable RTW ranged from months to years. Two participants were in the initial phase, and ten participants were in the post RTW 
Table 1. Disease-related characteristics of the participants. [+ denotes positive response; $C T=$ chemotherapy; RT=radiotherapy.]

\begin{tabular}{|c|c|c|c|c|c|c|c|c|c|c|c|}
\hline \multirow{3}{*}{$\begin{array}{l}\text { Partic- } \\
\text { ipant }\end{array}$} & \multirow{3}{*}{$\begin{array}{c}\text { Age } \\
\text { at inter- } \\
\text { view }\end{array}$} & \multirow{3}{*}{$\begin{array}{l}\text { Age } \\
\text { at diag- } \\
\text { nosis }\end{array}$} & \multirow{3}{*}{$\begin{array}{c}\text { Cancer } \\
\text { diagnosis }\end{array}$} & \multicolumn{6}{|c|}{ Treatment } & \multicolumn{2}{|c|}{ End of treatment } \\
\hline & & & & \multirow{2}{*}{$\begin{array}{l}\text { Sur- } \\
\text { gery }\end{array}$} & \multirow{2}{*}{ CT } & \multirow{2}{*}{$\mathrm{RT}$} & \multirow{2}{*}{ Hormone } & \multirow[t]{2}{*}{ Other } & \multirow[t]{2}{*}{ Duration of treatment } & \multirow{2}{*}{ 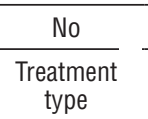 } & \multirow{2}{*}{$\begin{array}{c}\text { Yes } \\
\text { Time between end of } \\
\text { treatment and interview }\end{array}$} \\
\hline & & & & & & & & & & & \\
\hline $\bar{A}$ & 31 & 28 & Breast & + & + & & + & & $\begin{array}{l}6 \text { months and } 2 \text { years } \\
\text { of hormone treatment }\end{array}$ & Hormone & \\
\hline B & 50 & 47 & Breast & + & + & + & & & 7 months & & 2 years and 3 months \\
\hline \multirow[t]{3}{*}{ C } & 51 & 43 & Breast & + & + & + & & & Not recorded & & \\
\hline & & 48 & Local relapse & & + & + & & $\begin{array}{l}\text { Herceptin/ } \\
\text { Hyperthermia }\end{array}$ & Not recorded & & \\
\hline & & 50 & Lung metastasis & & + & & & & 2 months & CT & \\
\hline D & 51 & 44 & Breast & + & & + & + & & $\begin{array}{l}3 \text { months and } 5 \text { years of } \\
\text { hormone treatment }\end{array}$ & & 9 months \\
\hline E & 44 & 40 & Breast & + & + & + & + & $\begin{array}{l}\text { Herceptin/ } \\
\text { Ovariectomy }\end{array}$ & $\begin{array}{l}7 \text { months and } 2 \text { years of } \\
\text { hormone treatment }\end{array}$ & & 2 years \\
\hline $\mathrm{F}$ & 38 & 36 & Breast & + & + & + & & & 13 months & & 1 year and 6 months \\
\hline G & 41 & 40 & Breast & + & + & + & & & 5 months & CT/hormone & \\
\hline $\mathrm{H}$ & 47 & 44 & Breast & + & + & + & + & $\begin{array}{l}\text { Herceptin/ } \\
\text { Ovariectomy }\end{array}$ & $\begin{array}{l}12 \text { months and } 3 \text { years } \\
\text { of hormone treatment }\end{array}$ & Hormone & \\
\hline \multirow[t]{2}{*}{ I } & 37 & 28 & Breast & + & + & & + & & Not recorded & & \\
\hline & & 36 & $\begin{array}{l}\text { Lung, liver, bone } \\
\text { metastasis }\end{array}$ & & + & & + & & 6 months & Hormone & \\
\hline$J$ & 46 & 42 & Breast & + & + & + & & & 8 months & & 4 years \\
\hline K & 36 & 35 & Breast & + & & + & & & 4 months & & 1 year and 2 months \\
\hline $\mathrm{L}$ & 34 & 34 & Breast & + & + & & + & & 7 months & CT/hormone & \\
\hline
\end{tabular}

phase (table 2). For those participants who reached the sustainable RTW phase, an average RTW process could be divided into three phases: (i) during diagnosis and initial treatment, completely sick-listed for some time; (ii) during chemotherapy and/or radiotherapy, completely sick-listed but working on a flexible basis (hours, workplace, tasks) and with fewer responsibilities and reduced expectations of supervisor/colleagues (ie, phases 1 and 2 of initial RTW), and (iii) after radiotherapy or chemotherapy, gradual RTW, involving an increase in working hours, tasks, and responsibilities and gradually reducing paid sick leave (ie, post RTW). This pattern did not apply to four of the ten participants who were in their post RTW phase: one participant did not work through treatment, two participants had a new episode of sick leave after they had returned to work, and one participant was on disability pension due to cancer recurrence after she had returned to work (table 2).

\section{Factors that influence return to work}

Of the 12 participants, 9 experienced many barriers in their RTW process, involving all ICF domains (table 3). In contrast, only two participants reported no or minor problems and one participant reported that her work ability was improved due to her cancer experience. Par- ticipants mentioned key barriers in all domains, whereas key facilitators were found in environmental factors only (table 3 ). In addition, all participants were very motivated to resume work and all participants disclosed that they had cancer to their supervisor and colleagues.

Disease/disorder and body functions, body structures. Barriers to RTW include the disease itself, treatment, a slow or insufficient recovery over time, and having another co-morbidity. Participants mentioned slow or insufficient recovery over time as being caused by physical (eg, lymphoedema) and/or psychological side-effects (eg, concentration). As participant B recalled when trying to unlock a medicine cabinet at work: "During early return to work, it was also the confusion in my head. I was standing in front of the medicine cupboard, and then it already started with the login codes, they did not come to mind, I had to get the medicine."

Furthermore, temperament and personality functions, such as feeling guilty, feeling responsible or overly loyal, or having unrealistic expectations, were mentioned as barriers.

On the positive side, a recovery of breast cancer treatment, absence of long-term side effects, being confident and conscientious, and other positive temperament and personality functions were all mentioned as 
Table 2. Work-related characteristics of the participants. [RTW=return to work; Temp=temportary; Perm=permanent; Comm=communication.]

\begin{tabular}{|c|c|c|c|c|c|c|c|c|c|c|c|c|}
\hline \multirow{2}{*}{$\begin{array}{l}\text { Partic- } \\
\text { ipant }\end{array}$} & \multicolumn{5}{|c|}{ Work status before diagnosis } & \multicolumn{4}{|c|}{ Work status at time of interview } & \multirow{2}{*}{$\begin{array}{l}\text { Sick-listed } \\
\text { at time of } \\
\text { interview }\end{array}$} & \multirow{2}{*}{$\begin{array}{l}\text { RTW } \\
\text { character- } \\
\text { istics }\end{array}$} & \multirow{2}{*}{$\begin{array}{l}\text { Altered work } \\
\text { situation }\end{array}$} \\
\hline & Occupation & Contract & $\begin{array}{c}\text { Years } \\
\text { worked }\end{array}$ & $\begin{array}{l}\text { Work } \\
\text { hours }\end{array}$ & $\begin{array}{l}\text { Shift } \\
\text { work }\end{array}$ & Occupation & Contract & $\begin{array}{l}\text { Work } \\
\text { hours }\end{array}$ & $\begin{array}{l}\text { Shift } \\
\text { work }\end{array}$ & & & \\
\hline A & $\begin{array}{l}\text { Supervisor } \\
\text { call centre }\end{array}$ & Tem & 0.5 & 32 & Yes & $\begin{array}{l}\text { Re- } \\
\text { integration } \\
\text { counselor }\end{array}$ & Temp & 36 & No & No & $\begin{array}{l}\text { Worked through } \\
\text { treatment }\end{array}$ & $\begin{array}{l}\text { Improved occu- } \\
\text { pation, working } \\
\text { hours }\end{array}$ \\
\hline B & $\begin{array}{l}\text { Specialist } \\
\text { nurse }\end{array}$ & Perm & 30 & 32 & Yes & $\begin{array}{l}\text { Specialist } \\
\text { nurse }\end{array}$ & $\begin{array}{l}\text { Perm/ } \\
\text { partly } \\
\text { disability } \\
\text { pension }\end{array}$ & 24 & No & $\begin{array}{l}50 \% \\
\text { since } \\
\text { last week }\end{array}$ & $\begin{array}{l}\text { New episode of sick } \\
\text { leave }\end{array}$ & $\begin{array}{l}\text { Worsened work- } \\
\text { ing hours, work } \\
\text { content }\end{array}$ \\
\hline C & $\begin{array}{l}\text { Project } \\
\text { manager }\end{array}$ & Perm & 4 & 24 & No & Counsellor & $\begin{array}{l}\text { Disability } \\
\text { pension/ } \\
\text { freelance }\end{array}$ & Variable & No & $\begin{array}{l}\text { Disability } \\
\text { pension }\end{array}$ & $\begin{array}{l}\text { Worked through } \\
\text { treatment. }\end{array}$ & $\begin{array}{l}\text { Worsened job } \\
\text { loss }\end{array}$ \\
\hline D & Coach & Freelance & 5 & Variable & Yes & Counsellor & $\begin{array}{l}\text { Self- } \\
\text { employed }\end{array}$ & 24 & Yes & No & $\begin{array}{l}\text { Worked through } \\
\text { treatment }\end{array}$ & $\begin{array}{l}\text { Improved } \\
\text { flexible working } \\
\text { hours }\end{array}$ \\
\hline$E$ & $\begin{array}{l}\text { Super- } \\
\text { market } \\
\text { employee }\end{array}$ & Perm & 12 & 24 & No & Cashier & Perm & 24 & Yes & No & $\begin{array}{l}\text { Worked through } \\
\text { treatment }\end{array}$ & $\begin{array}{l}\text { Worsened work } \\
\text { content, shift } \\
\text { work, policy of } \\
\text { company }\end{array}$ \\
\hline $\mathrm{F}$ & $\begin{array}{l}\text { Comms } \\
\text { specialist }\end{array}$ & Perm & 8 & 40 & No & $\begin{array}{l}\text { Comms } \\
\text { specialist }\end{array}$ & Perm & 40 & No & No & $\begin{array}{l}\text { Worked through } \\
\text { treatment }\end{array}$ & Same \\
\hline G & $\begin{array}{l}\text { Project } \\
\text { manager }\end{array}$ & Perm & 9 & 34 & No & $\begin{array}{l}\text { Project } \\
\text { manager }\end{array}$ & Perm & 34 & No & $\begin{array}{l}100 \% \\
\text { since } \\
\text { diagnosis }\end{array}$ & $\begin{array}{l}\text { Initial RTW phase, } \\
\text { working through } \\
\text { treatment }\end{array}$ & $\begin{array}{l}\text { Worsened } \\
\text { working hours }\end{array}$ \\
\hline $\mathrm{H}$ & $\begin{array}{l}\text { Sales } \\
\text { manager }\end{array}$ & Perm & 2 & 32 & No & $\begin{array}{l}\text { Sales } \\
\text { manager }\end{array}$ & Perm & 32 & No & No & $\begin{array}{l}\text { Worked through } \\
\text { treatment }\end{array}$ & Same \\
\hline I & $\begin{array}{l}\text { Senior } \\
\text { policy } \\
\text { officer }\end{array}$ & Perm & 3.5 & 36 & Yes & $\begin{array}{l}\text { Senior } \\
\text { policy } \\
\text { officer }\end{array}$ & Perm & 36 & No & $\begin{array}{l}40 \% \\
\text { since last } \\
\text { diagnosis }\end{array}$ & $\begin{array}{l}\text { Worked through } \\
\text { treatment. New epi- } \\
\text { sode of sick leave }\end{array}$ & $\begin{array}{l}\text { Worsened } \\
\text { Working hours }\end{array}$ \\
\hline J & $\begin{array}{l}\text { Manager } \\
\text { hospital }\end{array}$ & Perm & 1.5 & 36 & No & $\begin{array}{l}\text { Policy } \\
\text { officer }\end{array}$ & Perm & 24 & No & No & $\begin{array}{l}\text { Worked through } \\
\text { treatment }\end{array}$ & $\begin{array}{l}\text { Worsened } \\
\text { working hours, } \\
\text { occupation }\end{array}$ \\
\hline K & $\begin{array}{l}\text { Project } \\
\text { manager }\end{array}$ & Perm & 3 & 32 & No & $\begin{array}{l}\text { Project } \\
\text { manager }\end{array}$ & Perm & 32 & No & $\begin{array}{l}33 \% \\
\text { since } 3 \\
\text { months }\end{array}$ & $\begin{array}{l}\text { Worked through } \\
\text { treatment. New epi- } \\
\text { sode of sick leave }\end{array}$ & $\begin{array}{l}\text { Worsened } \\
\text { working hours }\end{array}$ \\
\hline L & Taxi driver & Temp & 3 & 10 & No & Taxi driver & Temp & 10 & No & $\begin{array}{l}100 \% \text { since } \\
\text { diagnosis }\end{array}$ & $\begin{array}{l}\text { elnitial RTW phase. } \\
\text { No RTW }\end{array}$ & $\begin{array}{l}\text { Worsened job } \\
\text { loss }\end{array}$ \\
\hline
\end{tabular}

RTW facilitators. As participant A explained: "I really wanted to show them that I am not only [name of participant] with cancer, more than that, I am not that at all anymore."

Activity/participation. Participants indicated that negative physical and psychological side effects restricted them at the activity and/or participation level. For example, psychosocial complaints led to difficulties focusing attention and remembering facts, whereas lymphoedema impairments led to difficulties driving a car. Participant J pointed out: "Of course I have to do a lot of thinking [for my work]. Well, when that is disrupted in the beginning and you forget half of it, then that is another restriction."

Participants felt that they could either not perform at their pre-diagnosis level of work functioning or that they experienced difficulties doing other activities (eg, doing household tasks), which in turn decreased the possibilities for RTW.
At the activity and participation level, the following factors were identified as RTW facilitators: being economically self-sufficient, managing their employment (ie, re-education, seeking employment, maintaining a job, terminating a job), and taking care of their health. Participant F recalled: "I always tried to exercise in between [treatments] ... and you just notice that that is very good for your body but also for your mind ... Your mind resets so to speak ... and I think that it works very positively [for your work ability]."

Personal factors. On a personal level, there were several perceived barriers to RTW. Participants mentioned that they had to fight the stigma that work was not important to them during or after treatment, that they were not able to resume work, or that their work productivity was lower in comparison to healthy subjects. These stigmas became worse when diagnosed with metastatic cancer.

Many experienced cancer as a major life event that 
Table 3. Barriers/facilitators for return-to-work (RTW) classified according to the International Classification of Functioning, Disability and Health (ICF).

\begin{tabular}{|c|c|c|c|c|}
\hline Domain & Factor & $\begin{array}{l}\text { Barrier - } \\
\text { Facilitator + }\end{array}$ & $\begin{array}{l}\text { Initial * } \\
\text { Post ** } \\
\text { Both ***a }\end{array}$ & $\begin{array}{l}\text { Important } \\
\text { Barrier - } \\
\text { Facilitator }+{ }^{b}\end{array}$ \\
\hline Disease/ disorder & $\begin{array}{l}\text { Breast cancer, breast cancer treatment } \\
\text { Co-morbidity } \\
\text { (Slow or insufficient) recovery over time } \\
\text { Absence of breast cancer treatment side effects }\end{array}$ & $\begin{array}{l}- \\
- \\
-1+ \\
+\end{array}$ & $\begin{array}{l}* \star \star \\
\star \star \star \\
\star * \\
\star *\end{array}$ & - \\
\hline $\begin{array}{l}\text { Body functions/ body } \\
\text { structures }\end{array}$ & $\begin{array}{l}\text { Sensation of muscle stiffness } \\
\text { Having difficulties with mobility of joint functions } \\
\text { Lack of quality of sleep } \\
\text { Lack of functioning of immunological system functions } \\
\text { Fatiguability } \\
\text { Sensations associated with cardiovascular functions } \\
\text { Discomfort associated with menopause } \\
\text { Having difficulties with attention functions } \\
\text { Having difficulties with retrieval of memory } \\
\text { Having difficulties with pace of thought } \\
\text { Having difficulties with higher-level cognitive functions } \\
\text { Having difficulties applying knowledge } \\
\text { Having difficulties being confident } \\
\text { Conscientiousness } \\
\text { Confidence } \\
\text { Energy and drive functions } \\
\text { Temperament and personality functions, other specified }\end{array}$ & $\begin{array}{l}- \\
- \\
- \\
- \\
- \\
- \\
- \\
- \\
- \\
- \\
- \\
- \\
- \\
+ \\
+ \\
+ \\
-1+\end{array}$ & $\begin{array}{l}\star \\
\star \\
\star * \\
\star * \star \\
\star \star \star \\
\star \\
\star \\
\star \\
\star \\
\star \\
\star \\
\star \\
\star \\
\star \\
\star \star \star \\
\star * * \\
\star \star *\end{array}$ & - \\
\hline Activity/ participation & $\begin{array}{l}\text { Having difficulties focusing attention } \\
\text { Having difficulties driving } \\
\text { Having difficulties assisting others with self-care } \\
\text { Having difficulties doing housework } \\
\text { Looking after one's health } \\
\text { Vocational training } \\
\text { Seeking employment } \\
\text { Maintaining a job } \\
\text { Terminating a job } \\
\text { Economic self-sufficiency }\end{array}$ & $\begin{array}{l}- \\
- \\
- \\
- \\
+ \\
+ \\
+ \\
+ \\
+ \\
+\end{array}$ & $\begin{array}{l}\text { * } \\
\star \\
\star \\
\star \\
\star \star \star \\
\star \\
\star \star \\
\star \star \star \\
\star \star \star \\
\star \star \star\end{array}$ & $\begin{array}{l}- \\
-\end{array}$ \\
\hline Personal factors & $\begin{array}{l}\text { Struggle to fight stigmatization } \\
\text { Altered personality, priorities, or importance of work } \\
\text { (Having difficulties) coping } \\
\text { Job lock } \\
\text { Skills/competences }\end{array}$ & $\begin{array}{l}- \\
-/+ \\
-/+ \\
- \\
+\end{array}$ & $\begin{array}{l}* \star \star \\
\star * \star \\
\star * \star \\
\star \star \\
\star \star \star\end{array}$ & - \\
\hline $\begin{array}{l}\text { Environmental factors - } \\
\text { directly related to work }\end{array}$ & $\begin{array}{l}\text { Job content (physical and mental demands) } \\
\text { (Lack of) support from work environment } \\
\text { Negative/positive attitude of work environment } \\
\text { (Lesser degree of) control of working situation } \\
\text { Negative/positive terms of employment }\end{array}$ & $\begin{array}{l}-/+ \\
-/+ \\
-/+ \\
-/+ \\
-/+\end{array}$ & $\begin{array}{l}\star \star \star \\
\star \star \star \\
\star \star \star \\
\star \star \star \\
\star \star \star\end{array}$ & $\begin{array}{l}- \\
-/+ \\
-/+ \\
-/+ \\
-\end{array}$ \\
\hline $\begin{array}{l}\text { Environmental factors } \\
\text { - not directly related } \\
\text { to work }\end{array}$ & $\begin{array}{l}\text { Individual attitudes of health professionals } \\
\text { Societal attitudes } \\
\text { Invisibility of cancer, treatment, or long term side effects } \\
\text { Support and relationships (family) } \\
\text { Support and relationships (friends) } \\
\text { Support and relationships (personal assistants) } \\
\text { Support and relationships (health professionals) } \\
\text { Support and relationships (other professionals) } \\
\text { Support and relationships (other specified) } \\
\text { Social security services, systems and policies }\end{array}$ & $\begin{array}{l}- \\
- \\
- \\
+ \\
+ \\
+ \\
+/- \\
+ \\
+ \\
+\end{array}$ & $\begin{array}{l}\star \\
\star \star \star \\
\star \star \star \\
\star \star \star \\
\star \star \star \\
\star \\
\star \\
\star \star \star \\
\star \\
\star \star \star\end{array}$ & $\begin{array}{l}+ \\
+ \\
+\end{array}$ \\
\hline
\end{tabular}

a Influence of each factor during initial, post, or both RTW phases.

${ }^{b}$ Factors that were considered by participants as key barriers to or facilitators of RTW process. 
made them re-evaluate their work lives. For some, their social and family lives became a higher priority than before diagnosis. Others re-evaluated their personal work preferences in how they wanted to work and for whom. Some experienced difficulties coping with their cancer diagnosis and the RTW process because they found it difficult to ask help from others, explain their limitations to colleagues, set boundaries, or accept their limitations. Brought on by fear of recurrence, some participants reported that they did not dare to change jobs and reported a "job lock" because they either did not want to lose their permanent contract or employee benefits. As participant F explained: "Well, looking for a new job scares me a bit because I have a very good employer who treats you very well when you are ill, and when you accept an employment contract somewhere else, you never get a permanent employment contract straight away and imagine it [cancer] will come back, then you are in trouble."

Additionally many personal factors were perceived to be facilitators for RTW. Having various skills and competences, such as being able to fall back on work expertise, and having the skills to do more than the former tasks is believed to be a faciliator.

Equally, valuing the importance of work is a facilitator. All participants attributed various meanings to having paid work, including that work provides structure or distraction, it enables one to be economically selfsufficient and away from home, allows one to contribute to society, offers intellectual challenges, and represents a return to normalcy. As participant K stated: "Continuing working was definitely a way for me not to go crazy so to say, my whole life is arranged like that, I have my own job, I have a home, I am very independent."

A final personal factor that participants felt facilitates RTW is the implemention of coping strategies to deal with cancer and work, such as accepting limitations, being able to explain limitations, disclosing diagnosis and treatment, avoiding stress, accepting being fired, and being realistic towards supervisors and colleagues regarding one's own work capacity.

Environmental factors directly related to work. In this area, several barriers to RTW were noted. Job content including physical (eg, physical workload) and mental demands (eg, stressful job) can be problematic. Lack of support from colleagues, supervisors, employers, and/ or occupational physicians is also a barrier; many in the work environment do not know how to deal with cancer. This results in, on the one hand, insensitive management (eg, non-supportive work environment) or, on the other hand, over-protection. Participants noted also that they did not receive sufficient support from human resources or their company in general. In some cases, participants experienced negative attitudes about their work performance as cancer survivors or they felt the work environment overestimated their work functioning, resulting in unrealistic expectations or a lack of understanding of the long-term side effects. Furthermore, participants indicated that disclosure of cancer can evoke feelings of discomfort among colleagues and that cancer still remains a difficult subject to discuss at the workplace.

Not having control over the working situation (eg, the job can only be performed full-time or it is not possible to have a flexible work arrangement) is also perceived as a barrier as are negative terms of employment (eg, freelance work, being fired due to their illness). As participant L pointed out: "[After three temporary working contracts], I was going to get a permanent employment contract. Because I, yes, became ill and then they [employer] recognized, yes, we will just not do that ... Well the [employer] did not want to run the risk ... also because it takes too long, so to say, before you are back at your old level again."

On the positive side, environmental factors also play a role as facilitators of RTW. Having a job that was not physically demanding or where you can (temporarily) do undemanding tasks (job content) was viewed positively: "I temporarily did the easy things, such as marking down the products at the shop," explained participant E.

Support from subordinates, colleagues, supervisors, employers, and/or occupational physicians plays a key role in RTW. Support from supervisors consists, for example, of moderating someone's over-eagerness, taking away responsibilities, expressing confidence, and thinking along with the sick-listed employee. Support from colleagues and subordinates consists of social support, expressing confidence, giving positive feedback and encouragement, and making the cancer survivor feel that she has returned to a familiar and safe place. Support from the occupational physician consists of moderating someone's over-eagerness and making a RTW plan. Related to this, participants mentioned that a positive attitude in the work environment helped them. This includes understanding, having no expectations regarding productivity, and giving the employee the freedom to plan RTW as she wants. Like many environmental factors that can have a positive or negative influence depending on the situation, the upside of control over the working situation is having the ability to work from home or working flexibly (ie, plan one's day tasks, working hours flexibly throughout the week, and taking hours off when feeling ill). This can reduce the workload, which contributes to RTW.

Likewise positive terms of employment are reported as a facilitator because participants felt their employer wanted to keep them employed, offered them a new contract, or their job was being handled ably while there were on sick leave. 
Environmental factors - not directly related to work. There were many perceived RTW barriers outside of work. Individual attitudes of health professionals can be negative toward RTW; one participant mentioned that her oncological nurse had advised against returning to work in between radiotherapy and chemotherapy due to fatigue, even though she did not suffer from fatigue. Another mentioned that her occupational physician did not understand that she wanted to work during treatment, because she was able to stay at home and still receive $100 \%$ of her salary.

Societal attitudes are also important; participants described the general attitude that when you have cancer and undergo treatment, you are very ill and pitiful and thought of as not being able to work. On the other hand, there can be a certain "invisibility" of cancer, its treatment or long-term side effects: some stated that side effects such as fatigue and cognitive and emotional problems are not often visible, which can lead to less understanding at the workplace. Consequently, participants pointed out that they struggled with finding a balance between looking ill and being labeled as a cancer patient and, at the same time, receiving understanding and support. Therefore, some participants made a conscious choice to not wear a wig, so everyone could see that they had cancer and were ill as participant F explained: "Certainly, when you are bald from the chemo, you look ill. On the other hand, you don't look ill because of the medicine you get against nausea, which makes you rosy-cheeked, and then everybody cries: 'oh, you look so good' and that can be pretty frustrating ... The occupational physician once said, well ... better not to wear a wig because then they [colleagues] will see that you are ill ... otherwise there is a chance that you will be drawn into work too fast."

On the positive side, environmental factors not directly related to work can also be facilitators of RTW. Support and relationships with family, friends, personal assistants, health professionals, and other breast cancer survivors are important because they provide practical support and encouragement and enable discussion about RTW and being alone in a difficult situation. Participants reported mostly discussing work with their treating physician in general terms, but not in detail. Some mentioned being encouraged to go back to work or discussed a phased RTW. Other health professionals, such as an occupational physician specialized in cancer and work, a social worker, a psychologist, and a reintegration counselor, provided worthwhile support and advice. Rehabilitation improved bodily functions (eg, lymphoedema), which enhanced their possibilities to resume work. Finally, social security services, systems, and policies also facilitated RTW as participants noted that financial necessity, expiring sick leave pay, or the absence of a safety net forced them to resume work.

\section{Initial return to work}

The following factors that were described above were found during the initial RTW phase.

Physical and psychological side-effects. Participants mentioned that some physical and psychological sideeffects, such as cognitive-related side-effects, diminished over time and were only a hindering factor during initial RTW. Therefore, having difficulty concentrating, driving, taking care of their household, and taking care of their children were only a hindering factor during initial RTW.

Temporarily altered importance of work. Participants reported that they experienced a temporary altered importance of work that diminished mostly after returning to work full-time.

Altered work relationships. Some participants reported changes in work relationships on the one hand because colleagues go on with their normal lives and on the other hand because participants mentioned that they had changed fundamentally due to their cancer experience.

Other factors. During initial RTW, some temperament and personality functions, such as lacking confidence, were named as barriers, while being conscientious was a facilitator. In addition, the ability to re-educate was a facilitator during initial RTW. Finally, the advice received by one participant to not go back to work too soon was perceived as a barrier during initial RTW stage while peer support from other breast cancer survivors was mentioned as a facilitator.

\section{Post return to work}

The following factors that were described above were found during the post RTW phase.

Stigma. Colleagues tend to think that when a cancer survivor returns to work full-time and is not on sick leave anymore she is cured, fully recovered, and able to perform at her pre-diagnosis level. As a result, attention, understanding, and support gradually "wear off". In contrast, returning to work is seen by the participants as a process, not a singular event as their work environment may see it, and this difference in perception can lead to misunderstanding. Some participants reported having to deal with problems years after diagnosis or never being able to work at their pre-diagnosis level again. In addition, this different perception was, in some cases, reinforced by the paradoxical feeling of not wanting to be labeled as a cancer survivor and to be treated as normal but at the same time needing some understanding 
and support. This complicates the need for that support that they expect from their work environment.

Slow or insufficient recovery over time. A slow or insufficient recovery over time (eg, reduced quality of sleep, fatigue) was perceived as a barrier during post RTW phase.

Work-related factors. One participant reported that seeking a new job after she returned to her previous job was a facilitator during the post RTW phase. However, some participants reported a "job lock", which was perceived as a hindering factor.

\section{Possible solutions}

Some participants were unable to formulate solutions that could have helped improve their situation, while others mainly mentioned positive factors. Consequently, this topic was only discussed with six participants. The following possible solutions to RTW problems were put forward.

Improved guidance. More guidance from supervisors/ employers, occupational physicians, health professionals at the hospital, and re-integration counselors specialized in cancer were mentioned. A participant thought that making a clear comprehensive RTW plan that includes task, hours, and responsibilities is important; in this way, all stakeholders will be informed. Another participant stated that occupational physicians should have more knowledge about the cancer survivor's situation (eg, cancer treatment, work situation) and more awareness of the differences between cancer survivors regarding side-effects. Another participant stated that life after cancer (including work life) should be discussed at the end of treatment by a doctor or a breast cancer nurse. Finally, two participants mentioned that a re-integration counselor specialized in cancer should be added to the cancer care to provide comprehensive RTW guidance.

Improved information. Specific information for employers, human resources, and occupational physicians should be provided to enhance understanding about the possible side-effects of cancer treatment that can occur during initial diagnosis, treatment, and in the long-term. Especially, information about the fatigue fluctuation was mentioned. However, employers, human resources, and occupational physicians should also be well informed about the possibilities of returning to work during/after treatment and the fact that their work productivity can be as good as before diagnosis in order to diminish negative beliefs. Finally, participants mentioned that society should be informed about the fact that work can be important for cancer survivors and that they are able to resume work during or after treatment.

\section{Discussion}

The objective of this study was to identify factors experienced as barriers to and facilitators of the RTW of breast cancer survivors, which factors were important during the initial and post RTW phases, and possible solutions for breast cancer survivors' difficulties when returning to work. We found that participants experienced many barriers and facilitators in the RTW process, involving all ICF domains. Moreover, we found that participants mentioned key barriers in all domains, whereas key facilitators were only found in environmental factors. During initial RTW, physical and psychological side-effects hampered work resumption. In the post RTW stage, work resumption was hindered by the gradual "wearing-off" of understanding from the work environment. Participants mentioned that guidance by an occupational physician or healthcare professional should be improved and more information for the work environment should be available.

\section{Strengths and limitations}

Because of the qualitative nature of this study, these results need to be interpreted with caution and confirmed in a large and unbiased sample. The study population was a self-selected population who joined an online Dutch cancer patient organization, which may have resulted in the population consisting of young breast cancer survivors, who worked in the service sector, and who were very keen on returning to work (ie, our results may be biased by age, working sector, and motivation). Furthermore, a recall bias may be apparent, as initial experiences may be altered by later experiences. However, we tried to minimize this recall effect by asking participants to fill in a questionnaire before the start of the interview and by interviewing participants in different phases of their RTW process. Not all participants were able to identify possible solutions to problems they encountered. Additional studies are required, for which our results can be used as a starting point. The strengths of this study include the range of disease stages, treatment, and work situation of the population. Another strength is the use of the ICF, making the analysis of the interviews transparent. Because we first labeled all interviews with open codes, the possibility that our analysis was prejudiced by the description of the ICF is small. In addition, the use of the ICF makes it possible in the future to compare different (chronic) health conditions with each other.

\section{Comparison with the international literature}

For some themes, our results are comparable with previous qualitative studies concerning the RTW experiences 
of cancer survivors [eg, support from the work environment (4), work adaptations (18), importance of work (24), physical/psychological side-effects (18), re-evaluation of work life (18), and financial problems (18)]. However, new themes were found, including temperament and personality functions, job lock, taking care of one's health, skills/coping, societal attitudes, and the support of family and healthcare professionals.

In contrast to previous studies $(4,12,16,18)$, participants discussed RTW with their treating physician but did not receive detailed advice. However, they reported receiving worthwhile advice from an occupational physician specialized in cancer and work, a reintegration counselor, a psychologist, a social worker, or a rehabilitation programme. This finding indicates that other healthcare professionals play an important role in the RTW process and that interventions carried out by those professionals may be of benefit.

Contrary to another previous study carried out in the UK (13), we found that all participants were aware of occupational health services and that most participants received some form of support. It is likely that this discrepancy can be explained by the different social security systems of the UK and the Netherlands. Furthermore, we found, in contrast to previous studies $(6,18,25)$, that all participants disclosed their illness and treatment to their employer and colleagues. This difference may be explained by the fact that Dutch employees who are sick-listed due to cancer may have more confidence in their employer and the Dutch social security system, which may result in the notion that disclosing their illness may not have negative consequences. However, we found that some employers had negative illness beliefs about the duration of recovery needed and work capacity that resulted in the firing of an employee because of her illness. These negative beliefs may be enhanced by the disclosure of a cancer survivor about the time needed to return to work. Therefore, some employers should be better informed about the possibilities for cancer survivors to resume work to diminish negative illness beliefs (26).

Finally, in contrast to another study (25), we found that half of the participants changed their work. This anomoly may be explained by the fact that, in comparison to that study, participants in our study were more often treated with chemotherapy and/or radiotherapy, which in general leads to more difficulties for a cancer survivor and in turn may increase the necessity to alter their work.

\section{Implications for research and practice}

Most identified factors that were experienced as barriers to RTW were potentially modifiable. Because RTW patterns differed greatly among the participants and because participants experienced very diverse barriers to and facilitators of RTW, individual guidance seems most appropriate. However, some participants mentioned receiving worthwhile peer support from other breast cancer survivors, indicating that group support may offer an extra benefit. In addition, because different barriers are experienced during the initial and the post RTW process, an intervention should target different factors at different time points. Moreover, because most breast cancer survivors reported problems during both RTW phases, an intervention should be facilitated during their whole RTW process, including some substantial time after returning to work.

As most participants mentioned environmental factors as key facilitators for RTW, improving the knowledge, understanding, and awareness in the work environment is important for decreasing negative illness beliefs, but also for preventing a "wearing-off effect" of support and understanding and bringing attention to the importance of work for cancer survivors.

Finally, some participants reported having difficulties coping with their situation, accepting their limitations, and dealing with their unsupportive work environment. For those cancer survivors, learning how to cope with their difficulties may enhance RTW.

In conclusion, to improve RTW among cancer survivors, interventions should focus on barriers and facilitators for individuals at different time points in the process. Better guidance by healthcare professionals and information for the workplace could also lead to an increased and easier RTW for cancer survivors.

\section{Acknowledgement}

We would like to thank the cancer survivors for their participation. We would also like to thank the Dutch cancer patient organization (NFK), the Dutch breast cancer organization (BVN), and the Dutch breast cancer organization for young breast cancer survivors (Stichting Amazones) for the possibility to post a notice on their website. The study was supported by a grant from the Stichting Insituut Gak and is part of the research program "Pathways to work" (www.verbeteronderzoek.nl).

\section{References}

1. Roelen CA, Koopmans PC, van RW, Groothoff JW, van der Klink JJ, Bultmann U. Trends in return to work of breast cancer survivors. Breast Cancer Res Treat. 2011;128:237-42. http:// dx.doi.org/10.1007/s10549-010-1330-0.

2. Peugniez C, Fantoni S, Leroyer A, Skrzypczak J, Duprey M, Bonneterre J. Return to work after treatment for breast cancer: 
single center experience in a cohort of 273 patients. Bull Cancer. 2011;98:E69-E79.

3. Balak F, Roelen CA, Koopmans PC, Ten Berge EE, Groothoff JW. Return to work after early-stage breast cancer: a cohort study into the effects of treatment and cancer-related symptoms. J Occup Rehabil. 2008;18:267-72. http://dx.doi. org/10.1007/s10926-008-9146-z.

4. Amir Z, Neary D, Luker K. Cancer survivors' views of work 3 years post diagnosis: a UK perspective. Eur J Oncol Nurs. 2008;12:190-7. http://dx.doi.org/10.1016/j. ejon.2008.01.006.

5. Hansen JA, Feuerstein M, Calvio LC, Olsen CH. Breast cancer survivors at work. J Occup Environ Med. 2008;50:777-84. http://dx.doi.org/10.1097/JOM.0b013e318165159e.

6. Tiedtke C, de Rijk A, Dierckx de Casterle B, Christiaens MR, Donceel P. Experiences and concerns about 'returning to work' for women breast cancer survivors: a literature review. Psychooncology. 2010;19:677-83. http://dx.doi.org/10.1002/ pon.1633.

7. De Boer AGEM, Verbeek JHAM, Spelten ER, Uitterhoeve ALJ, Ansink AC, de Reijke TM, et al. Work ability and returnto-work in cancer patients. Br J Cancer. 2008;98:1342-7. http://dx.doi.org/10.1038/sj.bjc.6604302.

8. Taskila T, Lindbohm ML. Factors affecting cancer survivors' employment and work ability. Acta Oncol. 2007;46:446-51. http://dx.doi.org/10.1080/02841860701355048.

9. Spelten ER, Sprangers MAG, Verbeek JHAM. Factors reported to influence the return to work of cancer survivors: a literature review. Psychooncology. 2002;11:124-31. http:// dx.doi.org/10.1002/pon.585.

10. Earle CC, Chretien Y, Morris C, Ayanian JZ, Keating NL, Polgreen LA, et al. Employment among survivors of lung cancer and colorectal cancer. J Clin Oncol. 2010;28:1700-5. http://dx.doi.org/10.1200/JCO.2009.24.7411.

11. Spelten ER, Verbeek JH, Uitterhoeve AL, Ansink AC, van der Lelie J, de Reijke TM, et al. Cancer, fatigue and the return of patients to work-a prospective cohort study. Eur J Cancer. 2003;39:1562-7. http://dx.doi.org/10.1016/S09598049(03)00364-2.

12. Main DS, Nowels CT, Cavender TA, Etschmaier M, Steiner JF. A qualitative study of work and work return in cancer survivors. Psychooncology. 2005;14:992-1004. http://dx.doi. org/10.1002/pon.913.

13. Yarker J, Munir F, Bains M, Kalawsky K, Haslam C. The role of communication and support in return to work following cancer-related absence. Psychooncology. 2010;19:1078-85. http://dx.doi.org/10.1002/pon.1662.

14. World Health Organisation (WHO). International Classification of Functioning, Disability and Health (ICF). WHO: Geneva 2001.

15. Tong A, Sainsbury P, Craig J. Consolidated criteria for reporting qualitative research (COREQ): a 32-item checklist for interviews and focus groups. Int J Qual Health Care. 2007;19:349-57. http://dx.doi.org/10.1093/intqhe/mzm042.

16. Maunsell E, Brisson C, Dubois L, Lauzier S, Fraser A. Work problems after breast cancer: an exploratory qualitative study. Psychooncology. 1999;8:467-73. http://dx.doi. org/10.1002/(SICI)1099-1611(199911/12)8:6<467::AIDPON400>3.0.CO;2-P.

17. de Boer AGEM, van Zanten-Przybysz I, Maes LM, FringsDresen MH. Terugkeer naar werk na behandeling van kanker: ervaringen van patienten en leidinggevenden [Return to work after treatment for cancer: experiences of patients and supervisors]. TBV. 2008;16:292-7.

18. Kennedy F, Haslam C, Munir F, Pryce J. Returning to work following cancer: a qualitative exploratory study into the experience of returning to work following cancer. Eur J Cancer Care. 2007;16:17-25. http://dx.doi.org/10.1111/j.13652354.2007.00729.x.

19. Bradley CJ, Bednarek HL. Employment patterns of long-term cancer survivors. Psychooncology. 2002;11:188-98. http:// dx.doi.org/10.1002/pon.544.

20. Brach M, Cieza A, Stucki G, Fussl M, Cole A, Ellerin B, et al. ICF Core Sets for breast cancer. J Rehabil Med. 2004:121-7. http://dx.doi.org/10.1080/16501960410016811.

21. Escorpizo R, Ekholm J, Gmunder HP, Cieza A, Kostanjsek N, Stucki G. Developing a Core Set to describe functioning in vocational rehabilitation using the international classification of functioning, disability, and health (ICF). J Occup Rehabil. 2010;20:502-11. http://dx.doi.org/10.1007/s10926-0109241-9.

22. World Health Organization. ICF core set for vocational rehabilitation. 2010 [internet]. Accessed on August 2011. Available from: www.icf-research-branch.org/icf-core-setsprojects/diverse-situations/icf-core-sets-for-vocationalrehabilitation.html

23. Minis MA, Heerkens Y, Engels J, Oostendorp R, van EB. Classification of employment factors according to the International Classification of Functioning, Disability and Health in patients with neuromuscular diseases: A systematic review. Disabil Rehabil. 2009:1-14. http://dx.doi. org/10.1080/09638280902951838.

24. Peteet JR. Cancer and the meaning of work. Gen Hosp Psychiatry. 2000;22:200-5. http://dx.doi.org/10.1016/S01638343(00)00076-1.

25. Grunfeld EA, Cooper AF. A longitudinal qualitative study of the experience of working following treatment for gynaecological cancer. Psychooncology 2010 Nov 24. [Epub ahead of print]

26. Grunfeld EA, Low E, Cooper AF. Cancer survivors' and employers' perceptions of working following cancer treatment. Occup Med (Lond). 2010;60:611-7. http://dx.doi. org/10.1093/occmed/kqq143.

Received for publication: 30 June 2011 